\title{
State Estimation for Wireless Network Control System with Stochastic Uncertainty and Time Delay Based on Sliding Mode Observer
}

\author{
Pengfei Guo, ${ }^{1}$ Jie Zhang, ${ }^{1}$ Hamid Reza Karimi, ${ }^{2}$ Yurong Liu, ${ }^{3,4}$ Ming Lyu, ${ }^{5}$ and Yuming Bo \\ ${ }^{1}$ School of Automaton, Nanjing University of Science \& Technology, Nanjing 210094, China \\ ${ }^{2}$ Department of Engineering, Faculty of Engineering and Science, University of Agder, 4898 Grimstad, Norway \\ ${ }^{3}$ Department of Mathematics, Yangzhou University, Yangzhou 225002, China \\ ${ }^{4}$ Faculty of Engineering, King Abdulaziz University, Jeddah 21589, Saudi Arabia \\ ${ }^{5}$ Information Overall Department, North Information Control Group Co., Ltd., Nanjing 211153, China
}

Correspondence should be addressed to Jie Zhang; zhangjie.njust@gmail.com

Received 31 January 2014; Revised 17 April 2014; Accepted 22 April 2014; Published 7 May 2014

Academic Editor: Shuping He

Copyright (C) 2014 Pengfei Guo et al. This is an open access article distributed under the Creative Commons Attribution License, which permits unrestricted use, distribution, and reproduction in any medium, provided the original work is properly cited.

\begin{abstract}
State estimation problem is considered for a kind of wireless network control system with stochastic uncertainty and time delay. A sliding mode observer is designed for the system under the situation that no missing measurement occurs and system uncertainty happens in a stochastic way. The observer designed for the system can guarantee the system states will be driven onto the sliding surface under control law, and the sliding motion of system states on sliding surface will be stable. By constructing proper LyapunovKrasovskii functional, sufficient conditions are acquired via linear matrix inequality. Finally, simulation result is employed to show the effectiveness of the proposed method.
\end{abstract}

\section{Introduction}

During the past decades, state estimation problem is a hot issue in academical field, and researchers made fruitful research on both theoretical research and practical applications [1-4]. At the same time, research and applications in wireless network control system (WiNCS) attracted much interest from scholars [5-9]. Compared with wired network control system, WiNCS is more convenient in integrated wiring and could be arranged in hazardous area people cannot easily reach. Sensor nodes are distributed in advance to collect information and transmit signal back via signal channel through multihop technology, until they reach users' terminal applications. Because of its low cost, mobility character, and convenience for using, WiNCS is widely used in hospital monitoring, military and urban affairs, and some other important cases [10-13]. However, as the whole system is becoming complicated and integrated, people want to keep track of useful measured values, and a rich body of literature has appeared on the topic of state estimation or observerbased design; see [14-16] and the references therein.

As a special class of complex networks, WiNCS has its own characteristics due mainly to large numbers of nodes distributed over the region of interest. In this case, sensor nodes may be in mobile motion, which is attributed to the uncertainty in system state, and much effort has been made on this issue [17-22]. For distributed state estimation problem, each sensor node in WiNCS estimates system state not only from its own measurement but also from its neighboring nodes, so some unpredictable factors which happen in a stochastic way will enhance the complexity in state estimation [23-25]. Reference [26] studied synchronization problem for Markovian jump neural networks with time-varying delay and variable samplings. Stochastic stability of the error system was guaranteed by two criteria, and mode-independent controller was designed based on the maximum sampling interval. Reference [27] constructed proper Lyapunov-Krasovskii functional and studied the adaptive fault estimation problems 
for stochastic Markovian jump systems (MJSs), combined with time delays. Adaptive fault detection observer was designed and the sufficient condition was proposed. Reference [28] concentrated on the proportional-integral control problems of stochastic Markovian jump systems (MJSs) with uncertain parameters. It transformed the controller design problem to an output feedback control problem, and a sufficient condition was proposed via LMI.

Nodes receive measurement information from other nodes in WiNCS which is time consuming, so time delay cannot be avoided in the research of WiNCS, and many scholars devoted to this problem [29-33]. Reference [34] considered the problem that both had discrete and distributed delays, and the delay dependent passivity condition was acquired. Reference [35] investigated the problem of sampled-data extended dissipative control for uncertain Markov jump systems. By proposing a new integral inequality, a novel exponential stability criterion and an extended dissipativity condition were established. In addition, a sufficient condition for desired mode-independent sampled-data controller was also obtained. Reference [36] dealt with the average consensus problem in directed networks of agents with switching topology and time delay. It proved that all the agents could reach average consensus under a proper time delay if the topology of agents was weakly connected and balanced.

Motivated by previous research stated above, our target is to deal with state estimation problem in wireless network control system, which contains stochastic uncertainty and time delay. A sliding mode observer is designed in two steps; by the use of Lyapunov stability theory, sufficient conditions are proposed to make sure that system states can be driven onto the sliding surface within finite time and make stable sliding motion on sliding surface.

The rest of the paper is organized as follows. In Section 2, the state estimation problem of WiNCS is formulated and some useful lemmas are introduced. In Section 3, we make our designing process in two steps. Besides, the gain of observer is acquired by LMI. An illustrated example is given in Section 4 to demonstrate the effectiveness of the proposed method. Finally, we give our conclusions in Section 5.

\section{Problem Formulation}

In this paper, we consider the following discrete stochastic and time delay model:

$$
\begin{aligned}
x(k+1) & =\left(A+\alpha_{k} \Delta A\right) x(k)+A_{d} \sum_{i=1}^{N} x(k-i)+B u(k), \\
y(k) & =C x(k),
\end{aligned}
$$

where $x(k) \in \mathbb{R}^{n}$ is the system state vector, $y(k) \in \mathbb{R}^{n}$ is the measured output, $\Delta A$ is internal perturbation arising from uncertain factors, $u(k)$ is system input, $A, A_{d}, B$, and $C$ are constant matrices with appropriate dimensions, and $\alpha_{k}$ is Bernoulli distributed white sequences governed by

$$
\begin{aligned}
& \operatorname{Prob}\left\{\alpha_{k}=1\right\}=\mathbb{E}\left\{\alpha_{k}\right\}=\alpha, \\
& \operatorname{Prob}\left\{\alpha_{k}=0\right\}=1-\mathbb{E}\left\{\alpha_{k}\right\}=1-\alpha,
\end{aligned}
$$

where $\alpha \in[0,1]$ is a known constant.

Remark 1. Most nodes in WiNCS may be in dynamic motion; they collect information from areas of interest. However, they may be affected by external environment, such as temperature, humidity, and topography, or linkage status inside the system, so system uncertainty may happen in a stochastic way, which increases the complexity of the system.

We make the following assumptions for system model (1).

Assumption 2. The perturbation parameter of the system satisfies

$$
\Delta A=G D(k) H .
$$

Respectively, $G$ and $H$ are known constant matrix, $D(k)$ is time-delay uncertain matrix, yet Lebesgue-measurable, and $D^{k}(t) D(k) \leq I$.

Assumption 3. $C^{T} C$ is full rank.

Assumption 4. All the states of the system can be measured and no missing measurement occurs.

We construct the following sliding mode observer:

$$
\begin{aligned}
\widehat{x}(k+1)= & A \widehat{x}(k)+A_{d} \sum_{i=1}^{N} \widehat{x}(k-i) \\
& +B u(k)+L[y(k)-\widehat{y}(k)]+w(k), \\
\widehat{y}(k)= & C \hat{x}(k),
\end{aligned}
$$

where $L$ is the gain of observer to be designed and $w(k)$ is nonlinear item in observer.

So the state error $e_{x}(k)$ and output error $e_{y}(k)$ of the system are as follows:

$$
\begin{aligned}
& e_{x}(k)=x(k)-\widehat{x}(k), \\
& e_{y}(k)=y(k)-\widehat{y}(k) .
\end{aligned}
$$

In this case, the system error model is

$$
\begin{aligned}
e_{x}(k+1)= & (A-K C) e_{x}(k)+\left(\alpha_{k}-\alpha\right) \Delta A x(k) \\
& +\alpha \Delta A x(k)+A_{d} \sum_{i=1}^{N} e_{x}(k-i)-w(k), \\
e_{y}(k+1)= & C e_{x}(k) .
\end{aligned}
$$

We define the sliding surface $s(k)$ as

$$
s(k)=e_{y}(k) \text {. }
$$


Our target in this paper is to find the gain of observer $L$ and the nonlinear input $w(k)$ that can drive system state onto the sliding surface within finite time and, in addition, make sure that sliding motion of system states on sliding surface is stable. Two conditions should be satisfied when designing sliding mode observer [37]:

(1) system error model is asymptotically stable when $s(k+1)=s(k)=0$;

(2) sliding mode manifold satisfies $\|s(k+1)\|<\|s(k)\|$.

Remark 5. We set system output error as sliding surface; the benefit is, condition (1) guarantees that sliding motion on sliding surface is stable, and condition (2) ensures that system state can be driven onto sliding surface within finite time. In this case, we convert state estimation problem into sliding mode observer design problem.

Besides, some useful and important lemmas that will be used in deriving out results are introduced below.

Lemma 6. Let $Y=Y^{T}, D, E$, and $F(t)$ be real matrix of proper dimensions, and $F^{T}(t) F(t) \leq I$; then inequality $Y+D F E+$ $(D F E)^{T}<0$ holds if there exists a constant $\varepsilon$, which makes the following equation hold:

$$
Y+\varepsilon D D^{T}+\varepsilon^{-1} E^{T} E<0 .
$$

Lemma 7 (Schur complement). Given a symmetric matrix $S=\left[\begin{array}{ll}S_{11} & S_{12} \\ S_{21} & S_{22}\end{array}\right]$, where $S_{11}$ is $r \times r$ dimensional, the following three conditions are equivalent:

(1) $S<0$;

(2) $S_{11}<0, S_{22}-S_{12}^{T} S_{11}^{-1} S_{12}<0$;

(3) $S_{22}<0, S_{11}-S_{12} S_{22}^{-1} S_{12}^{T}<0$.

Lemma 8. For any $x, y \in R^{n}, \mu>0$, the following equation holds:

$$
2 x^{T} y \leq \mu x^{T} x+\frac{1}{\mu} y^{T} y .
$$

\section{Main Results}

In this section, two theorems will be given. The first one ensures that system states can reach the sliding surface within finite time.

Theorem 9. For system error model (6) which meets Assumptions 2 , 3, and 4, and $w(k)=w_{1}(k)+w_{2}(k)$, where $w_{1}(k)=$ $A_{d} \sum_{i=1}^{N} e_{x}(k-i), w_{2}(k)=G H x(k)$, system states will be driven onto the sliding surface within finite time if there exists a general matrix $L \in R^{n_{x} \times n_{y}}$ making (10) hold:

$$
\left[\begin{array}{cc}
-C^{T} C & \sqrt{3+\alpha}(A-L C)^{T} C^{T} \\
* & -I
\end{array}\right]<0
$$

Proof. Considering $s(k+1)=e_{y}(k+1)=C e_{x}(k+1)$ and $\|s(k+1)\|<\|s(k)\|$, we have

$$
\begin{aligned}
& \mathbb{E}\left\{s^{T}(k+1) s(k+1)-s^{T}(k) s(k)\right\} \\
&= \mathbb{E}\left\{e_{y}^{T}(k+1) e_{y}(k+1)-e_{y}^{T}(k) e_{y}(k)\right\} \\
&= e_{x}^{T}(k)\left[(A-L C)^{T} C^{T} C(A-L C)-C^{T} C\right] e_{x}(k) \\
&+2 \alpha e_{x}^{T}(k)(A-L C)^{T} C^{T} C \Delta A x(k) \\
&+2 e_{x}^{T}(A-L C)^{T} C^{T} C A_{d} \sum_{i=1}^{N} e_{x}(k-i) \\
&+2 \alpha x^{T}(k) \Delta A^{T} C^{T} C A_{d} \sum_{i=1}^{N} e_{x}(k-i) \\
&+2 e_{x}^{T}(k)(A-L C)^{T} C^{T} C w(k) \\
&+2 \sum_{i=1}^{N} e_{x}^{T}(k-i) A_{d}^{T} C^{T} C w(k) \\
&+2 \alpha x^{T}(k) \Delta A^{T} C^{T} C w(k)+\alpha x^{T}(k) \Delta A^{T} C^{T} C \Delta A x(k) \\
&+\sum_{i=1}^{N} e_{x}^{T}(k-i) A_{d}^{T} C^{T} C \sum_{i=1}^{N} e_{x}(k-i)+w^{T}(k) C^{T} C w(k) .
\end{aligned}
$$

According to Lemma 8, we have

$$
\begin{aligned}
2 \alpha e_{x}^{T}(k) & (A-L C)^{T} C^{T} C \Delta A x(k) \\
\leq & \alpha e_{x}^{T}(k)(A-L C)^{T} C^{T} C(A-L C) e_{x}(k) \\
& +\alpha x^{T}(k) \Delta A^{T} C^{T} C \Delta A x(k), \\
2 e_{x}(k)^{T}(A-L C)^{T} C^{T} C A_{d} \sum_{i=1}^{N} e_{x}(k)(k-i) & \\
\leq & e_{x}(k)^{T}(A-L C)^{T} C^{T} C(A-L C) e_{x}(k) \\
& +\sum_{i=1}^{N} e_{x}(k)^{T}(k-i) A_{d}^{T} C^{T} C A_{d} \sum_{i=1}^{N} e_{x}(k)(k-i), \\
2 \alpha x^{T}(k) & \Delta A^{T} C^{T} C A_{d} \sum_{i=1}^{N} e_{x}(k-i) \\
\leq & \alpha x^{T}(k) \Delta A^{T} C^{T} C \Delta A x(k) \\
& +\alpha \sum_{i=1}^{N} e_{x}^{T}(k-i) A_{d}^{T} C^{T} C A_{d} \sum_{i=1}^{N} e_{x}(k-i),
\end{aligned}
$$




$$
\begin{aligned}
2 e_{x}^{T}(k) & (A-L C)^{T} C^{T} C w(k) \\
\leq & e_{x}^{T}(k)(A-L C)^{T} C^{T} C(A-L C) e_{x}(k) \\
& +w^{T}(k) C^{T} C w(k), \\
2 \sum_{i=1}^{N} e_{x}^{T}(k-i) A_{d}^{T} C^{T} C w(k) & \\
\leq & \sum_{i=1}^{N} e_{x}^{T}(k-i) A_{d}^{T} C^{T} C A_{d} \sum_{i=1}^{N} e_{x}(k-i)+w^{T}(k) C^{T} C w(k),
\end{aligned}
$$

$$
\begin{aligned}
& 2 \alpha x^{T}(k) \Delta A^{T} C^{T} C w(k) \\
& \quad \leq \alpha x^{T}(k) \Delta A^{T} C^{T} C \Delta A x(k)+\alpha w^{T}(k) C^{T} C w(k) .
\end{aligned}
$$

Substituting (12) to (17) into (11), we have

$$
\begin{aligned}
& \mathbb{E}\left\{s^{T}(k+1) s(k+1)-s^{T}(k) s(k)\right\} \\
& \leq e_{x}^{T}(k)\left[(3+\alpha)(A-L C)^{T} C^{T} C(A-L C)-C^{T} C\right] e_{x}(k) \\
&+x^{T}(k)\left[(3+\alpha) \Delta A^{T} C^{T} C \Delta A\right] x(k) \\
& \sum_{i=1}^{N} e_{x}^{T}(k-i)\left[(3+\alpha) A_{d}^{T} C^{T} C A_{d}^{T}\right] \\
& \times \sum_{i=1}^{N} e_{x}(k-i)+(3+\alpha) w^{T}(k) C^{T} C w(k) \\
&=e_{x}^{T}(k)\left[(3+\alpha)(A-L C)^{T} C^{T} C(A-L C)-C^{T} C\right] e_{x}(k) \\
&+ {\left[\Delta A x(k)-w_{2}(k)\right]^{T}(3+\alpha) C^{T} C\left[\Delta A x(k)-w_{2}(k)\right] } \\
&+ {\left[A_{d} \sum_{i=1}^{N} e_{x}(k-i)-w_{1}(k)\right]^{T} } \\
& \times(3+\alpha) C^{T} C\left[A_{d} \sum_{i=1}^{N} e_{x}(k-i)-w_{1}(k)\right] \\
& \leq e_{x}^{T}(k)\left[(3+\alpha)(A-L C)^{T}\right. \\
& \times(3+\alpha) C^{T} C\left[A_{d} \sum_{i=1}^{N} e_{x}(k-i)-w_{1}(k)\right] . \\
& \times\left[G H x(k)-w_{2}(k)\right]^{T}(3+\alpha) C^{T} C\left[G H x(k)-w_{2}(k)\right] \\
&+ {\left[A_{d} \sum_{i=1}^{N} e_{x}(k-i)-w_{1}(k)\right]^{T} } \\
&\left.+L C)-C^{T} C\right] e_{x}(k)
\end{aligned}
$$

We make $w_{1}(k)=A_{d} \sum_{i=1}^{N} e_{x}(k-i), w_{2}(k)=G H x(k)$, so we have

$$
\begin{aligned}
\mathbb{E} & \left\{s^{T}(k+1) s(k+1)-s^{T}(k) s(k)\right\} \\
& \leq e_{x}^{T}(k)\left[(3+\alpha)(A-L C)^{T} C^{T} C(A-L C)-C^{T} C\right] e_{x}(k) \\
& \leq 0 .
\end{aligned}
$$

By Schur complement, inequality (19) is equivalent to

$$
\left[\begin{array}{cc}
-C^{T} C & \sqrt{3+\alpha}(A-L C)^{T} C^{T} \\
* & -I
\end{array}\right]<0 .
$$

The proof of Theorem 9 is complete.

Next, we will prove that sliding motion on sliding surface is stable.

Theorem 10. For system error model (6) which meets Assumptions 2, 3, and 4, and $w(k)=w_{1}(k)+w_{2}(k)$, where $w_{1}(k)=$ $A_{d} \sum_{i=1}^{N} e_{x}(k-i), w_{2}(k)=G H x(k)$, sliding motion of system states on sliding surface will be stable if there exists a general matrix $L \in R^{n_{x} \times n_{y}}$ and $P \in R^{n_{x} \times n_{x}}$ making (21) hold:

$$
\left[\begin{array}{cc}
-P & \sqrt{3+\alpha}(A-L C)^{T} P \\
* & -P
\end{array}\right]<0
$$

Proof. We construct the following Lyapunov-Krasovskii functional:

$$
V(k)=e_{x}^{T}(k) P e_{x}(k) .
$$

Making difference of $V(k)$, we have

$$
\begin{aligned}
\mathbb{E}\left\{e_{x}^{T}(k+1) P e_{x}(k+1)-e_{x}^{T}(k) P e_{x}(k)\right\} \\
=e_{x}^{T}(k)\left[(A-L C)^{T} P(A-L C)-P\right] e_{x}(k) \\
\quad+2 \alpha e_{x}^{T}(k)(A-L C)^{T} P \Delta A x(k) \\
+2 e_{x}^{T}(A-L C)^{T} P A_{d} \sum_{i=1}^{N} e_{x}(k-i) \\
+2 \alpha x^{T}(k) \Delta A^{T} P A_{d} \sum_{i=1}^{N} e_{x}(k-i) \\
+2 e_{x}^{T}(k)(A-L C)^{T} P w(k) \\
+2 \sum_{i=1}^{N} e_{x}^{T}(k-i) A_{d}^{T} P w(k)+2 \alpha x^{T}(k) \Delta A^{T} P w(k) \\
+\alpha x^{T}(k) \Delta A^{T} P \Delta A x(k) \\
+\sum_{i=1}^{N} e_{x}^{T}(k-i) A_{d}^{T} P \sum_{i=1}^{N} e_{x}(k-i)+w^{T}(k) P w(k) .
\end{aligned}
$$


According to Lemma 8, we have

$$
\begin{aligned}
& 2 \alpha e_{x}^{T}(k)(A-L C)^{T} P \Delta A x(k) \\
& \leq \alpha e_{x}^{T}(k)(A-L C)^{T} P(A-L C) e_{x}(k) \\
& \quad+\alpha x^{T}(k) \Delta A^{T} P \Delta A x(k), \\
& 2 e_{x}(k)^{T}(A-L C)^{T} P A_{d} \sum_{i=1}^{N} e_{x}(k)(k-i) \\
& \leq e_{x}(k)^{T}(A-L C)^{T} P(A-L C) e_{x}(k) \\
& \quad+\sum_{i=1}^{N} e_{x}^{T}(k-i) A_{d}^{T} P A_{d} \sum_{i=1}^{N} e_{x}(k-i) \\
& 2 \alpha x^{T}(k) \Delta A^{T} P A_{d} \sum_{i=1}^{N} e_{x}(k-i) \\
& \leq \alpha x^{T}(k) \Delta A^{T} P \Delta A x(k) \\
& \quad+\alpha \sum_{x}^{N}(k)(A-L C)_{x}^{T}(k-i) A_{d}^{T} P A_{d} \sum_{i=1}^{N} e_{x}(k-i) \\
& \quad(k)(A-L C)^{T} P w(k) \\
& \quad(k) e_{x}(k)+w^{T}(k) P w(k),
\end{aligned}
$$

$$
\begin{aligned}
& 2 \sum_{i=1}^{N} e_{x}^{T}(k-i) A_{d}^{T} P w(k) \\
& \quad \leq \sum_{i=1}^{N} e_{x}^{T}(k-i) A_{d}^{T} P A_{d} \\
& \quad \times \sum_{i=1}^{N} e_{x}(k-i)+w^{T}(k) P w(k), \\
& 2 \alpha x^{T}(k) \Delta A^{T} P w(k) \\
& \leq \alpha x^{T}(k) \Delta A^{T} P \Delta A x(k)+\alpha w^{T}(k) P w(k) .
\end{aligned}
$$

$$
\begin{aligned}
& \leq e_{x}^{T}(k)\left[(3+\alpha)(A-L C)^{T} P(A-L C)-P\right] e_{x}(k) \\
& +\left[G H x(k)-w_{2}(k)\right]^{T}(3+\alpha) P\left[G H x(k)-w_{2}(k)\right] \\
& +\left[A_{d} \sum_{i=1}^{N} e_{x}(k-i)-w_{1}(k)\right]^{T} \\
& \quad \times(3+\alpha) P\left[A_{d} \sum_{i=1}^{N} e_{x}(k-i)-w_{1}(k)\right] .
\end{aligned}
$$

We make $w_{1}(k)=A_{d} \sum_{i=1}^{N} e_{x}(k-i), w_{2}(k)=G H x(k)$, so we have

$$
\begin{aligned}
& \mathbb{E}\left\{e_{x}^{T}(k+1) P e_{x}(k+1)-e_{x}^{T}(k) P e_{x}(k)\right\} \\
& \quad \leq e_{x}^{T}(k)\left[(3+\alpha)(A-L C)^{T} P(A-L C)-P\right] e_{x}(k) \\
& \quad \leq 0 .
\end{aligned}
$$

By Schur complement again, inequality (31) is equivalent to

$$
\left[\begin{array}{cc}
-P & \sqrt{3+\alpha}(A-L C)^{T} P \\
* & -P
\end{array}\right]<0 .
$$

The proof of Theorem 10 is complete.

Substituting (24) to (29) into (23), we have

$$
\begin{aligned}
& \mathbb{E}\left\{e_{x}^{T}(k+1) P e_{x}(k+1)-e_{x}^{T}(k) P e_{x}(k)\right\} \\
& \leq e_{x}^{T}(k)\left[(3+\alpha)(A-L C)^{T} P(A-L C)-P\right] e_{x}(k) \\
& \quad+x^{T}(k)\left[(3+\alpha) \Delta A^{T} P \Delta A\right] x(k) \\
& \sum_{i=1}^{N} e_{x}^{T}(k-i)\left[(3+\alpha) A_{d}^{T} P A_{d}^{T}\right] \\
& \quad \times \sum_{i=1}^{N} e_{x}(k-i)+(3+\alpha) w^{T}(k) P w(k)
\end{aligned}
$$

\section{Numerical Simulations}

In this section, a numerical simulation is given for testing the theorems developed in this paper. Consider the system model (1), where

$$
\begin{aligned}
A & =\left[\begin{array}{ccc}
6.18 & 0 & -3 \\
5.64 & 1.005 & 3.15 \\
2.97 & 0 & 3.3
\end{array}\right], \\
A_{d} & =\left[\begin{array}{ccc}
-3.38 & 0.15 & 0 \\
-0.285 & 0.93 & 0.48 \\
-0.555 & 1.17 & 2.235
\end{array}\right], \\
C & =\left[\begin{array}{lll}
2.8800 & 1.1048 & 0.0150 \\
1.3095 & 0.7680 & 0.0195 \\
0.0930 & 0.0315 & 1.0800
\end{array}\right], \quad B=\left[\begin{array}{c}
1.2 \\
0.7 \\
1
\end{array}\right],
\end{aligned}
$$



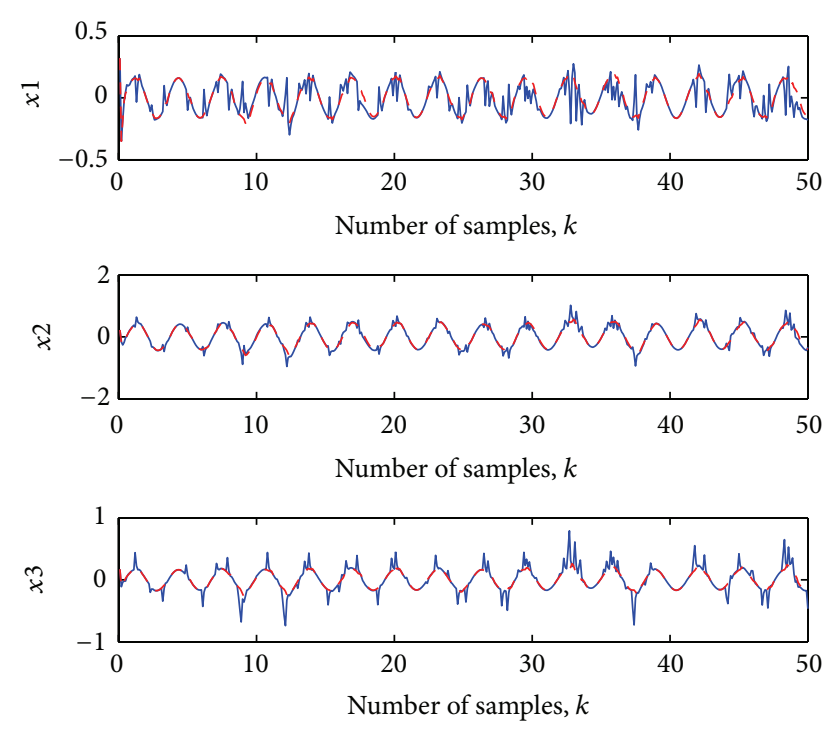

FIGURE 1: Trajectories of system states and observed value.

$$
\begin{aligned}
& G=\left[\begin{array}{ccc}
0.1 & 0.2 & -0.1 \\
0 & 0.23 & -0.76 \\
0.87 & 0.5 & -0.32
\end{array}\right], \\
& H=\left[\begin{array}{ccc}
0.184 & 0.112 & 0.23 \\
0.097 & -0.16 & -0.156 \\
-0.277 & -0.069 & -0.152
\end{array}\right] \text {, } \\
& D(k)=\left[\begin{array}{ccc}
0.8 \sin (0.7 k) & 0 & 0 \\
0 & 0.8 \sin (0.7 k) & 0 \\
0 & 0 & 0.8 \sin (0.7 k)
\end{array}\right] \text {, } \\
& u(k)=\sin (0.3 k), \quad \alpha=0.15 \text {. }
\end{aligned}
$$

The initial states are $x(k)=\left[\begin{array}{ll}1.3 & 0.427-0.92\end{array}\right]^{T}$; according to Theorems 9 and 10, we have

$$
\begin{aligned}
& L=\left[\begin{array}{lll}
6.3100 & -8.9663 & -2.7035 \\
3.8252 & -4.3148 & 2.9414 \\
2.8593 & -4.2400 & 3.0924
\end{array}\right], \\
& P=10^{5} \times\left[\begin{array}{ccc}
1.3987 & 0 & 0 \\
0 & 1.3987 & 0 \\
0 & 0 & 1.3987
\end{array}\right] .
\end{aligned}
$$

Simulation results are shown in Figure 1; red line denotes real states value and blue line denotes observed value. From the figure, we can see that the estimated value tracks the real value very well, which can fully demonstrate the effectiveness of the proposed method. States error is shown in Figure 2, which is much smaller than observer design method proposed in [38], shown in Figure 3; this is because slide mode control is insensitive to uncertainty and external disturbance. However, we can see from Figure 2 that when system states are at turning point, states error becomes larger; this is because chattering is inevitable in slide mode control, which is our future work.
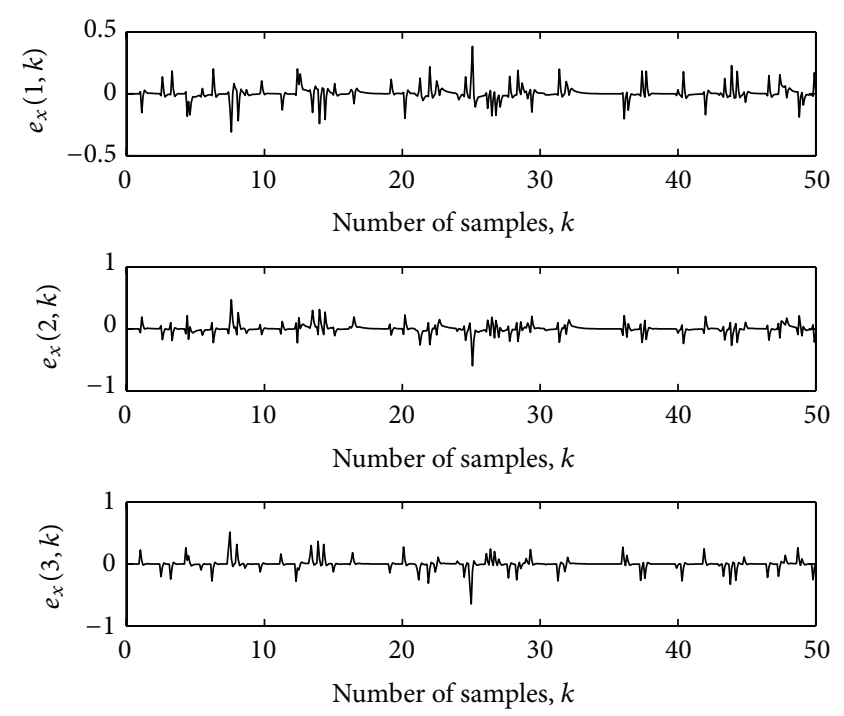

FIGURE 2: Trajectories of system states error.
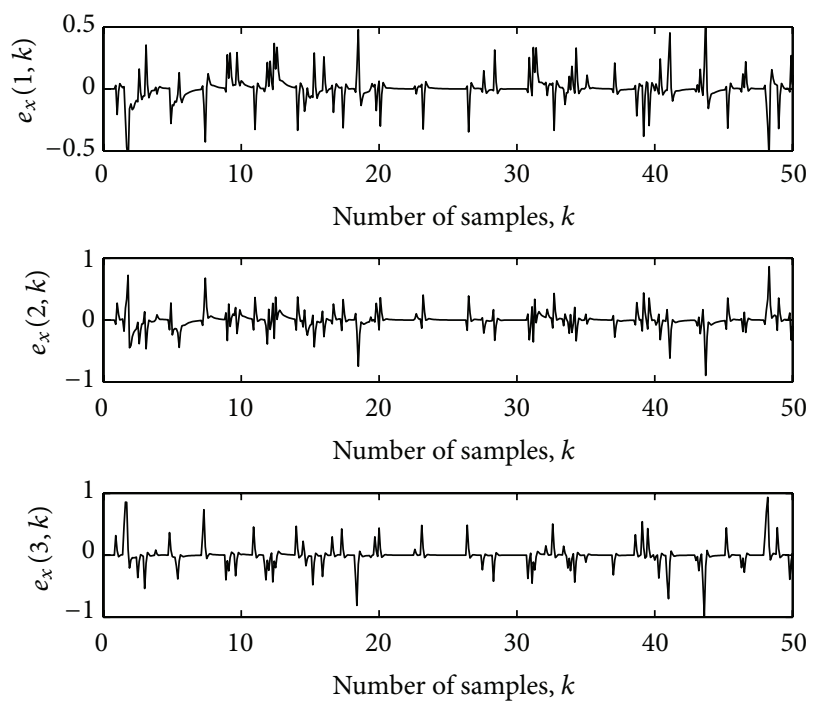

FIGURE 3: Trajectories of system states error by applying method proposed in [38].

\section{Conclusion}

This paper considers state estimation problem in wireless network control system with stochastic uncertainty and time delay. System uncertainty is assumed to occur in a stochastic way, which increases system complexity, and time delay is also considered. A sliding mode observer is designed in two steps; by constructing proper Lyapunov-Krasovskii functional, sufficient condition is acquired via linear matrix inequality. Finally, simulation result is employed to show the effectiveness of the proposed method.

\section{Conflict of Interests}

The authors declare that there is no conflict of interests regarding the publication of this paper. 


\section{Acknowledgments}

This work has been supported by the National Natural Science Foundation of China (Grant no. 61104109), the Natural Science Foundation of Jiangsu Province of China (Grant no. BK2011703), the Support of Science and Technology and Independent Innovation Foundation of Jiangsu Province of China (Grant no. BE2012178), and the Doctoral Fund of Ministry of Education of China (Grant no. 20113219110027).

\section{References}

[1] J. Liang, Z. Wang, and X. Liu, "Distributed state estimation for uncertain Markov-type sensor networks with mode-dependent distributed delays," International Journal of Robust and Nonlinear Control, vol. 22, no. 3, pp. 331-346, 2012.

[2] L. Wu, P. Shi, and H. Gao, "State estimation and slidingmode control of Markovian jump singular systems," IEEE Transactions on Automatic Control, vol. 55, no. 5, pp. 1213-1219, 2010.

[3] O. Kosut, L. Jia, R. J. Thomas, and L. Tong, "Malicious data attacks on smart grid state estimation: attack strategies and countermeasures," in Proceedings of the First IEEE International Conference on Smart Grid Communications (SmartGridComm '10), pp. 220-225, 2010.

[4] B. Shen, Z. Wang, and X. Liu, "Bounded $H_{\infty}$ synchronization and state estimation for discrete time-varying stochastic complex networks over a finite horizon," IEEE Transactions on Neural Networks, vol. 22, no. 1, pp. 145-157, 2011.

[5] H. R. Karimi and H. Gao, "New delay-dependent exponential $H_{\infty}$ synchronization for uncertain neural networks with mixed time delays," IEEE Transactions on Systems, Man, and Cybernetics B: Cybernetics, vol. 40, no. 1, pp. 173-185, 2010.

[6] G. W. Irwin, J. Chen, A. McKernan, and W. G. Scanlon, "Codesign of predictive controllers for wireless network control," IET Control Theory \& Applications, vol. 4, no. 2, pp. 186-196, 2010.

[7] H. R. Karimi and P. Maass, "Delay-range-dependent exponential $H_{\infty}$ synchronization of a class of delayed neural networks," Chaos, Solitons and Fractals, vol. 41, no. 3, pp. 1125-1135, 2009.

[8] D. Ding, Z. Wang, and B. Shen, "Recent advances on distributed filtering for stochastic systems over sensor networks," International Journal of General Systems, vol. 43, no. 3-4, pp. 372-386, 2014.

[9] H. R. Karimi, "Robust $H_{\infty}$ filter design for uncertain linear systems over network with network-induced delays and output quantization," Modeling, Identification and Control, vol. 30, no. 1, pp. 27-37, 2009.

[10] I. F. Akyildiz, X. Wang, and W. Wang, "Wireless mesh networks: a survey," Computer Networks, vol. 47, no. 4, pp. 445-487, 2005.

[11] D. Ding, Z. Wang, H. Dong, and H. Shu, "Distributed $H_{\infty}$ state estimation with stochastic parameters and nonlinearities through sensor networks: the finite-horizon case," Automatica, vol. 48, no. 8, pp. 1575-1585, 2012.

[12] J. Kay and J. Frolik, "Quality of service analysis and control for wireless sensor networks," in Proceedings of the IEEE International Conference on Mobile Ad-Hoc and Sensor Systems, pp. 359-368, October 2004.

[13] H. Dong, Z. Wang, D. W. C. Ho, and H. Gao, "Robust $H_{\infty}$ fuzzy output-feedback control with multiple probabilistic delays and multiple missing measurements," IEEE Transactions on Fuzzy Systems, vol. 18, no. 4, pp. 712-725, 2010.
[14] S. He and F. Liu, "Exponential passive filtering for a class of nonlinear jump systems," Journal of Systems Engineering and Electronics, vol. 20, no. 4, pp. 829-837, 2009.

[15] W. Ye, J. Heidemann, and D. Estrin, "Medium access control with coordinated adaptive sleeping for wireless sensor networks," IEEE/ACM Transactions on Networking, vol. 12, no. 3, pp. 493-506, 2004.

[16] S. He and F. Liu, "Observer-based finite-time control of timedelayed jump systems," Applied Mathematics and Computation, vol. 217, no. 6, pp. 2327-2338, 2010.

[17] H. R. Karimi, "A sliding mode approach to $H_{\infty}$ synchronization of master-slave time-delay systems with Markovian jumping parameters and nonlinear uncertainties," Journal of the Franklin Institute. Engineering and Applied Mathematics, vol. 349, no. 4, pp. 1480-1496, 2012.

[18] A. Micol, C. Martin, O. Dalverny, M. Mermet-Guyennet, and M. Karama, "Reliability of lead-free solder in power module with stochastic uncertainty," Microelectronics Reliability, vol. 49, no. 6, pp. 631-641, 2009.

[19] S. He, "Resilient $L_{2}-L_{\infty}$ filtering of uncertain markovian jumping systems within the finite-time interval," Abstract and Applied Analysis, vol. 2013, Article ID 791296, 7 pages, 2013.

[20] S. He and F. Liu, "Stochastic finite-time stabilization for uncertain jump systems via state feedback," Journal of Dynamic Systems, Measurement, and Control, vol. 132, no. 3, Article ID 034504, 2010.

[21] S. Peng, "Nonlinear expectations and stochastic calculus under uncertainty", Microelectronics Reliability, 2010, http://arxiv.org/abs/1002.4546.

[22] B. Shen, Z. Wang, H. Shu, and G. Wei, " $H_{\infty}$ filtering for uncertain time-varying systems with multiple randomly occurred nonlinearities and successive packet dropouts," International Journal of Robust and Nonlinear Control, vol. 21, no. 14, pp.16931709, 2011.

[23] H. R. Karimi, "Robust delay-dependent $H_{\infty}$ control of uncertain time-delay systems with mixed neutral, discrete, and distributed time-delays and Markovian switching parameters," IEEE Transactions on Circuits and Systems. I. Regular Papers, vol. 58, no. 8, pp. 1910-1923, 2011.

[24] Z. G. Wu, P. Shi, H. Su, and J. Chu, "Asynchronous $l_{2}-l_{\infty}$ filtering for discrete-time stochastic Markov jump systems with randomly occurred sensor nonlinearities," Automatica, vol. 50, no. 1, pp. 180-186, 2014.

[25] K. M. Hyde and H. R. Maier, "Distance-based and stochastic uncertainty analysis for multi-criteria decision analysis in excel using visual basic for applications," Environmental Modelling and Software, vol. 21, no. 12, pp. 1695-1710, 2006.

[26] Z. Wu, P. Shi, H. Su, and J. Chu, "Stochastic synchronization of markovian jump neural networks with time-varying delay using sampled data," IEEE Transactions on Cybernetics, vol. 43, no. 6, pp. 1796-1806, 2013.

[27] S. He and F. Liu, "Robust stabilization of stochastic Markovian jumping systems via proportional-integral control," Signal Processing, vol. 91, no. 11, pp. 2478-2486, 2011.

[28] S. He and F. Liu, "Robust stabilization of stochastic markovian jumping systems via proportional-integral control," Abstract and Applied Analysis, vol. 2012, Article ID 936375, 9 pages, 2012.

[29] S. He and F. Liu, "Unbiased estimation of markov jump systems with distributed delays," Signal Processing, vol. 100, pp. 85-92, 2014. 
[30] Z. Wang, Y. Liu, G. Wei, and X. Liu, "A note on control of a class of discrete-time stochastic systems with distributed delays and nonlinear disturbances," Automatica, vol. 46, no. 3, pp. 543-548, 2010.

[31] S. He and C. Liu, "Finite-time fuzzy control of nonlinear jump systems with time delays via dynamic observer-based state feedback," IEEE Transactions on Fuzzy Systems, vol. 20, no. 4, pp. 605-614, 2012.

[32] J. Sun, G. P. Liu, J. Chen, and D. Rees, "Improved delay-rangedependent stability criteria for linear systems with time-varying delays," Automatica, vol. 46, no. 2, pp. 466-470, 2010.

[33] P. Lin and Y. Jia, "Average consensus in networks of multi-agents with both switching topology and coupling time-delay," Physica A: Statistical Mechanics and its Applications, vol. 387, no. 1, pp. 303-313, 2008.

[34] Z. G. Wu, P. Shi, H. Su, and J. Chu, "Passivity analysis for discrete-time stochastic markovian jump neural networks with mixed time delays," IEEE Transactions on Neural Networks, vol. 22, no. 10, pp. 1566-1575, 2011.

[35] H. Shen, J. H. Park, L. Zhang, and Z. Wu, "Robust extended dissipative control for sampled-data markov jump systems," International Journal of Control, pp. 1-16, 2013.

[36] J. Qin, H. Gao, and W. X. Zheng, "Second-order consensus for multi-agent systems with switching topology and communication delay," Systems \& Control Letters, vol. 60, no. 6, pp. 390-397, 2011.

[37] C. Edwards, S. K. Spurgeon, and R. J. Patton, "Sliding mode observers for fault detection and isolation," Automatica, vol. 36, no. 4, pp. 541-553, 2000.

[38] W. Chen and S. Mehrdad, "Observer design for linear switched control systems," in Proceedings of the American Control Conference, vol. 6, pp. 5796-5801, July 2004. 


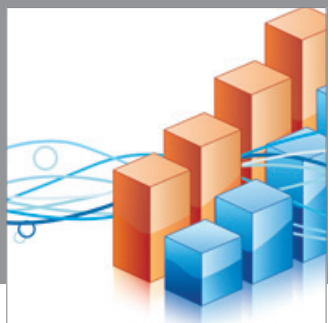

Advances in

Operations Research

mansans

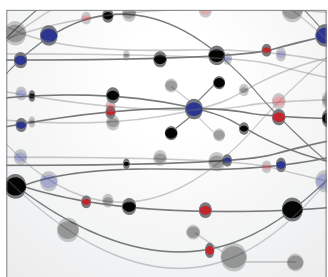

The Scientific World Journal
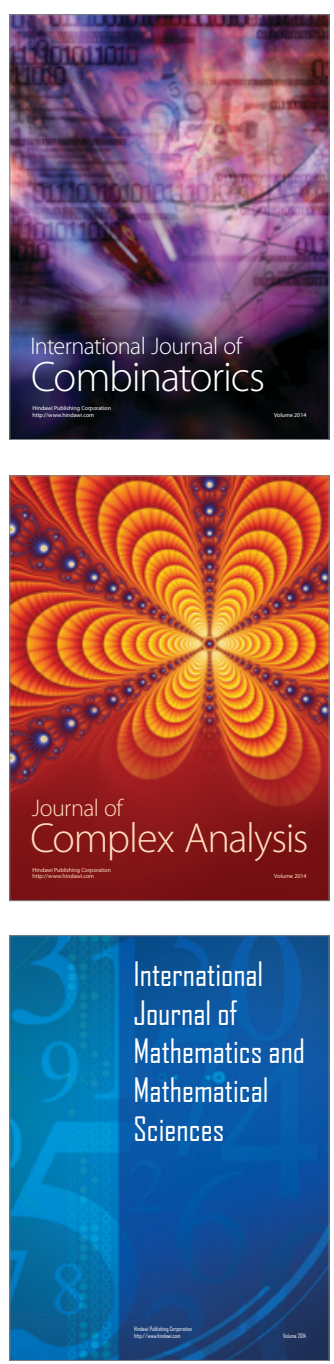
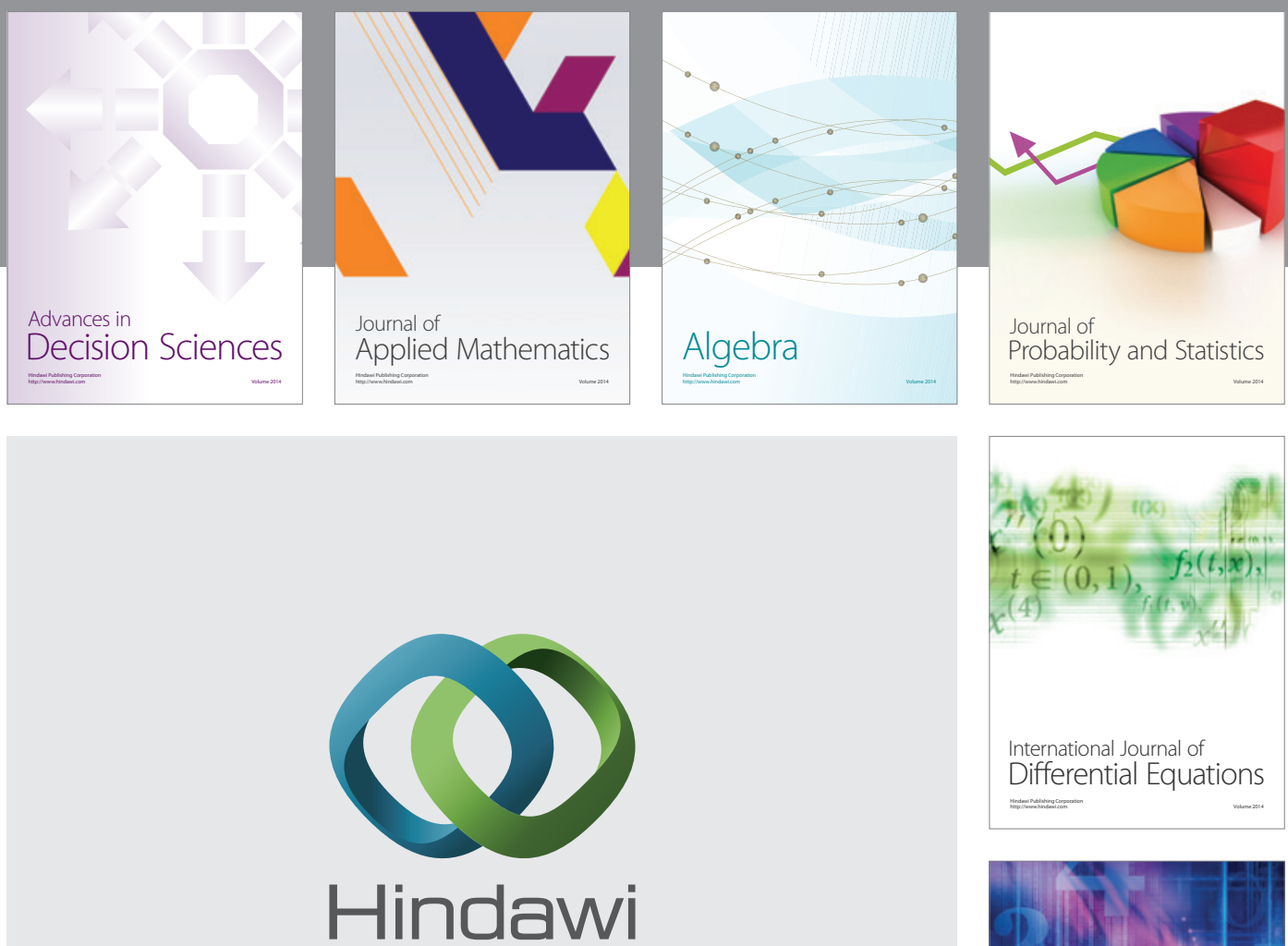

Submit your manuscripts at http://www.hindawi.com
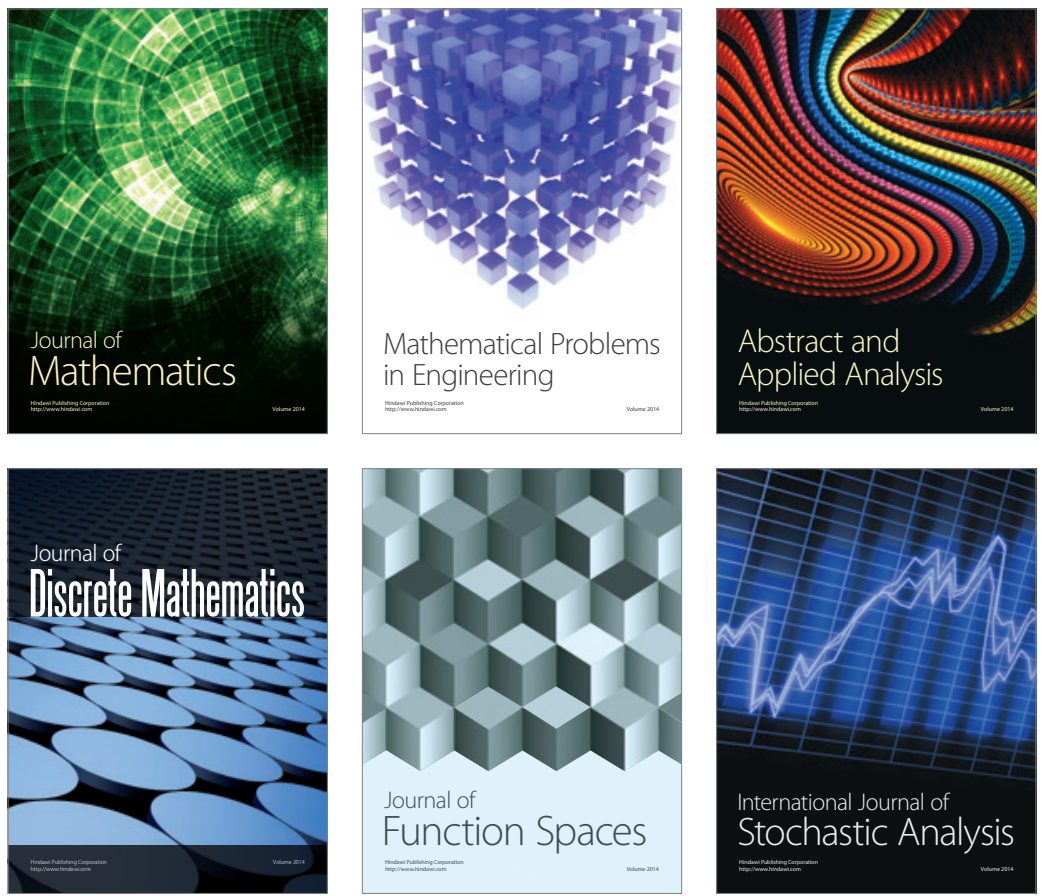

Journal of

Function Spaces

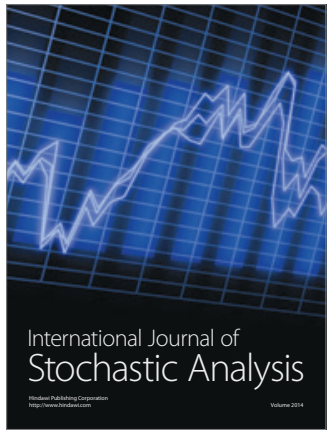

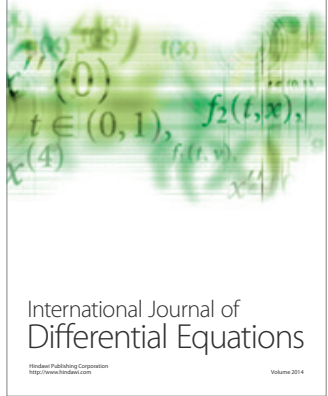
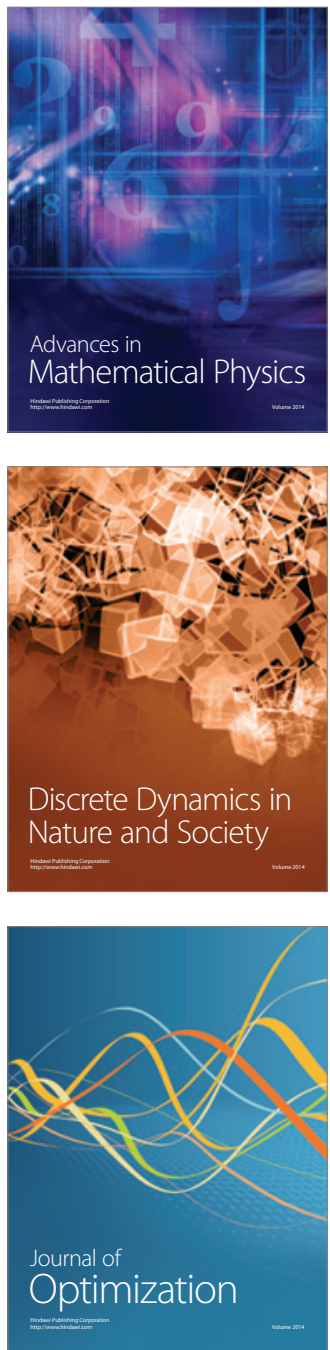